\title{
Zwischen Integration und »westlicher « Emanzipation: Verfassungsrechtliche Perspektiven zum Kopftuch(-verbot) und der Gleichberechtigung
}

\section{Das Kopftuch als multidimensionales Problem}

Das Kopftuch hat in den vergangenen Jahren nicht nur in Deutschland ${ }^{1}$ für viel Diskussionsstoff gesorgt. Vor fünf Jahren hat der Rechtsstreit der Kopftuch tragenden Lehrerin Fehresta Ludin begonnen, die auf ihre Einstellung in den Lehrdienst in Baden-Württemberg klagte und durch alle Instanzen bis hin zum Bundesverfassungsgericht ging. Seitdem ist hierzulande eine fast unüberschaubare Zahl von juristischen, politik-, sozial- und kulturwissenschaftlichen Beiträgen und Büchern und eine noch viel größere Menge an Zeitungs- und Zeitschriftenartikeln erschienen, die sich mit dem »Kopftuch « beschäftigen ${ }^{2}$. Aus verfassungsrechtlicher Perspektive geht es auf den ersten Blick um einen klassischen Grundrechtskonflikt: Es ist das Spannungsverhältnis zwischen individuellem Freiheitsanspruch der muslimischen Lehrerin und zweckgerichteter staatlicher Freiheitsbegrenzung durch die Schulbehörde. Die Vielschichtigkeit, ja Multidimensionalität des verhandelten Themas wird erst auf den zweiten Blick sichtbar. Da geht es dann um die Frage nach der Sichtbarkeit von Religion oder Religiosität in einem vom »Staat« beherrschten Bereich, um das historisch beladene und schwierige Verhältnis zwischen »Religion« und »Staat« im Allgemeinen ${ }^{3}$. Was bedeutet in diesem Zusammenhang »staatliche Neutralität«? Folgen wir einem integrierenden Neutralitätsbegriff, der die religiöse Lebensäußerung der Beamtin toleriert und vom Staat lediglich verlangt, sich nicht auf die Seite einer bestimmten Glaubensrichtung zu stellen (Nichtidentifikation) ${ }^{4}$ ? Oder hegen wir strengere Neutralitätsvorstellungen bis hin zu einer vollständigen Ausblendung von Glaubensüberzeugungen in der »staatlichen Gemeinschaft « im Sinne französischer »laicité « ${ }^{5}$ ?

Auch mit dem Hinweis auf die Frage nach der Neutralität ist dann jedoch nicht alles gesagt. Das »Kopftuch« wirft auch Fragen nach dem Umgang mit Religionen auf, denen nicht die Mehrheitsgesellschaft, sondern denen eingewanderte »Minderheiten « anhängen. Was bedeutet das Kopftuch in einer Gesellschaft, die - jedenfalls in Westdeutschland - bis in die 70er Jahre von christlichen Konfessionen dominiert war, sich aber heute einem zunehmenden Pluralismus von Glaubensrichtungen gegenüber sieht $^{6}$ ? Hier versteckt sich das Problem kultureller Fremdheit ${ }^{7}$, die Frage nach dem

1 S. Markard/Baer, (K)ein deutsches Problem: Religiöse Kleidung vor Gericht - Ein internationaler Vergleich, in: Haug/Reimer (Hg.), Politik ums Kopftuch, 2005, S. 151 ff.

2 Einen Überblick über die Diskussion gibt Oestreich, Der Kopftuch-Streit, 2004, S. 35 ff.

3 Aus verfassungsrechtlicher Perspektive s. Kloepfer, DÖV 2006, 45 ff.

4 In diesem Sinne: BVerfGE 108, S. 283 (303 ff.).

5 S. Gusy, KritV 2004, S. 153 (157 ff.); zum franzöischen Verständnis des Laizismus s. auch den Artikel von Jane Kramer, Taking the Veil, in: The New Yorker, November 2, 2004, S. 59.

6 Das thematisieren u.a. Heinig/Morlok, JZ 2003, S. 777, Häußler, ZAR 2004, S. 6; s. auch Kleff, Die unvollendete Säkularisierung und der Islam in Deutschland, in: Haug/Reimer, Politik ums Kopftuch, 2005, S. 85; einseitig hingegen: Bertrams, DVBl. 2003, S. 1225.

7 S. Britz, KJ 2003, S. 95. 
Verhältnis der »westlichen« Gesellschaft zum Islam ${ }^{8}$ oder Fragen nach den Wegen und den möglichen Versäumnissen bei der Integration der »Einwanderer « und »Gastarbeiter « in Mitteleuropa ${ }^{9}$ und nicht zuletzt nach der Furcht vor allseits bewusster Segregation und religiösem Fundamentalismus ${ }^{10}$.

Betrachten wir schließlich das Kopftuch als ein Kleidungsstück, das religiöse Inhalte zum Ausdruck bringt, also auch einen symbolischen Charakter hat, dann öffnet sich eine weitere Dimension: Das Kopftuch ist ein Zeichen, das in einen andauernden Kommunikationsprozess ${ }^{11}$ eingebettet ist und mit sehr unterschiedlichen Bedeutungen aufgeladen wird. Hier geht es entscheidend um die Frage, wer in diesem kommunikativen Austausch die Definitionsmacht besitzt, also letztlich bestimmen darf, wie das »muslimische Kopftuch« richtig zu deuten ist.

\section{Geschlecht - ein »blinder Fleck«}

Neben zahlreichen Aspekten, die also in der Kopftuchdebatte ineinander greifen, spielt die Kategorie »Geschlecht« oder genauer »Gender« eine besondere Rolle. Das Kopftuch wird von vielen als ein Ausdruck der Geschlechterhierarchie und der Unterdrückung von Frauen im Islam angesehen. Nicht zuletzt hat Alice Schwarzer, eine bekannte Protagonistin »der « Frauenbewegung in der Bundesrepublik, sich vehement gegen die Tolerierung des Kopftuchs im Schuldienst ausgesprochen. Das Kopftuch ist für sie die »Flagge des islamischen Kreuzzugs, der die ganze Welt zum Gottesstaat deformieren will ${ }^{12}$. Fehresta Ludin sei mit ihrer Forderung, das Kopftuch auch im Schuldienst zu tragen, eine Handlangerin des fundamentalistischen Islam, der weltweit die Unterdrückung der Frauen betreibe ${ }^{13}$. Die Entscheidung des Bundesverfassungsgerichts, das ein Kopftuch im Schuldienst nicht generell verbieten will, ist für Schwarzer bereits ein $»$ halber Sieg für die Fanatiker ${ }^{14}$.

Viele Feministinnen nehmen einen ähnlichen Standpunkt ein ${ }^{15}$. Andere zeichnen ein völlig kontrastierendes Bild: In ihrem Manifest für »Religiöse Vielfalt statt Zwangsemanzipation! - Aufruf wider eine Lex Kopftuch«, der von Marieluise Beck, Barbara

8 Bergmann, ZAR 2004, S. 135 (136).

9 U.a. Langenfeld, RdJB 2004, S. 4; s. auch Rhode, Der Islam - Alltagskonflikte und Lösungen, 2. Aufl. 2001.

10 S. Bergmann, ZAR 2004, S. 135 (136).

11 S. Oebbecke, Das »islamische Kopftuch« als Symbol, in: Festschrift für Rüffner, 2003, S. 593 (595).

12 Schwarzer, Herablassende Pseudo-Toleranz - Kein Schleier im Namen von Gleichheit und Selbstbestimmung, in: Frankfurter Rundschau, 02.06.2003, S. 8.

13 Schwarzer, Ludin - die Machtprobe, Emma Juli/August 2003, www.emma.de.

14 Schwarzer, Ein halber Sieg für die Fanatiker - Über das Kopftuch-Urteil in Karlsruhe, in: Haug/Reimer (Hg.), Politik ums Kopftuch, 2005, S. 32.

15 S. den Brief von Bendowski, Sander, Langer, der von etwa 100 meist prominenten Feministinnen unterzeichnet wurde, wiedergegeben von Haug, Der Kopftuchstreit als politisches Lehrstück, in: Haug/Reimer (Hg.), Politik ums Kopftuch, 2005, S. 171 (174f.); typisch auch: Pfeiffer, Das Kopftuch muss zu Hause bleiben - Ein frauenpolitischer Standpunkt, VR 2004, S. 415. 
John und Rita Süssmuth initiiert wurde, weisen die Unterzeichnerinnen darauf hin, dass »das Verbot des Kopftuchs für Lehrerinnen die allgemeine gesellschaftliche Stigmatisierung derjenigen Frauen, die es tragen, vorantreibt «. Es müsse vielmehr darum gehen, »muslimische Frauen auf ihrem Berufsweg zu stärken und es ihnen möglich zu machen, einen selbstbewussten, frei gewählten Lebensentwurf zu verfolgen « ${ }^{16}$.

So tritt die Bedeutung der Kategorie »Geschlecht « in bestimmten politischen Auseinandersetzungen also offen zu Tage. In der juristischen Debatte bleibt sie dagegen weitgehend unterbelichtet. Nun sind juristische Diskurse dadurch geprägt, dass sie bestimmte Argumente und Blickwinkel bewusst ausblenden, weil diese »vor dem Gesetz « nicht relevant sind. Jedoch ist die Gleichberechtigung hinsichtlich des Geschlechts im Grundgesetz in Art. 3 Abs. 2 und 3 verankert. In Absatz 2 heißt es: »Männer und Frauen sind gleichberechtigt. Der Staat fördert die tatsächliche Durchsetzung der Gleichberechtigung von Frauen und Männern und wirkt auf die Beseitigung bestehender Nachteile hin «. Absatz 3 verbietet die Benachteiligung oder Bevorzugung einer Person u.a. »wegen des Geschlechts«. Insofern ist es schon überraschend, dass die Frage nach der Bewertung des Kopftuchs unter diesem Gesichtspunkt - bis auf wenige Ausnahmen ${ }^{17}$ - in der juristischen Literatur so wenig Beachtung gefunden hat ${ }^{18}$. Es ist ein $»$ blinder Fleck $\aleph^{19}$, der gefüllt zu werden verdient. Er ließe sich differenziert im Rahmen des europarechtlichen Antidiskriminierungsrechts ${ }^{20}$ bearbeiten ${ }^{21}$. Da dessen Vorgaben in Deutschland noch nicht umgesetzt sind, konzentrieren wir uns auf das Verfassungsrecht.

\section{Kopftuchverbot als Diskriminierung muslimischer Frauen?}

Das Grundrecht auf Gleichstellung von Männern und Frauen lässt sich im Kopftuchstreit für beide Seiten ins Feld führen. Wird dem Kopftuch eine antiemanzipatorische, teilweise sogar fundamentalistische Bedeutung beigemessen, ist dies mit dem Verfassungsauftrag zur Gleichstellung nicht vereinbar ${ }^{22}$. Damit ist die dogmatische Funktion des Art. 3 Abs. 2 GG als Schutz- oder Fördergebot bzw. als kollidierendes Verfas-

16 Beck/ John u.a., »Religiöse Vielfalt statt Zwangsemanzipation!« - Aufruf wider eine Lex Kopftuch, abgedruckt in: Haug/Reimer (Hg.), Politik ums Kopftuch, 2005, S. 10 (11).

17 S. etwa Britz, KJ 2003, S. 95.

18 In der Entscheidung des Bundesverfassungsgerichts wird diese Frage nur an einer Stelle angesprochen, und zwar im Minderheitsvotum, s. BVerfGE 108, 282, 314 (333f.).

19 Oestreich, Der Kopftuch-Streit, 2004, S. 77.

20 Einschlägig ist insb. die Richtlinie zur Gleichbehandlung von Männern und Frauen 76/207/ EWG, die durch die Richtlinie 2002/72/EG dem aktuellen Stand europäischen Antidiskriminierungsrechts angepasst wurde. Art. 14 EMRK verbietet die Ungleichbehandlung aufgrund des Geschlechts im Anwendungsbereich der Konventionsrechte, dazu EGMR, NJW 2001, S. 2871 (2873) - Dhalab; das weiter gehende 12. Zusatzprotokoll zur EMRK, das eine Diskriminierung aufgrund des Geschlechts generell verbietet, hat die Bundesrepublik bis dato (noch) nicht ratifiziert.

21 Baer/Wrase, DÖV 2005, S. 243 (251).

22 So u.a. die dissentierenden Richter des BVerfGE 108, S. 282 (332 ff.); ähnlich z.B. Hufen, NVwZ 2004, S. 575 (576); Battis/Bultman, JZ 2004, S. 581 (583). 
sungsrecht angesprochen, die ein staatliches Verbot des Kopftuchs rechtfertigen kann. Auf der anderen Seite - und das wird häufig übersehen - streitet Art. 3 Abs. 2, Abs. 3 GG aber auch zugunsten von Muslima, die das Kopftuch tragen. Dazu lässt sich unterschiedlich argumentieren.

\section{a) Die Bedeutung von Art. $3 G G$}

Art. 3 Abs. 2 Satz 1 und Abs. 3 Satz 1 GG wurden lange nur als Differenzierungsverbote verstanden. Danach darf das Geschlecht einer Person - außer den vom BVerfG engen Ausnahmefällen ${ }^{23}$ - kein Maßstab für eine gesetzliche Unterscheidung sein. Diese formale Gleichheitsbetrachtung wird heute durch eine materiale Prüfung ergänzt. Danach müssen staatliche Maßnahmen auch dann gerechtfertig werden, wenn sie an scheinbar »neutrale « Kriterien anknüpfen, in der Lebenswirklichkeit allerdings Männer oder Frauen unterschiedlich betreffen ${ }^{24}$. In der US-amerikanischen Rechtsprechung ist das als Verbot des »disparate impact « seit langem judiziert, in Europa oder auch im Arbeitsrecht als Ungleichbehandlung durch mittelbare Diskriminierung anerkannt.

Für den Fall des Kopftuchs in der Schule ist dies unmittelbar relevant. Einschlägige Landesgesetze sind so formuliert, dass sie sämtliche religiösen Symbole und Kleidungsstücke erfassen, also auch solche, die Männer tragen ${ }^{25}$, wie etwa die jüdische Kippa oder den Turban eines $\mathrm{Sikh}^{26}$. Diese Landesgesetze knüpfen also weder direkt an das Geschlecht an noch an ein damit in zwingendem Zusammenhang stehendes Merkmal. Dann liegt nach früherer Ansicht keine Diskriminierung vor. Wird aber differenzierter analysiert, ob eine Regelung auch tatsächlich »neutral « ist, also auch gleiche Wirkung entfaltet, könnte der Befund anders aussehen.

Im Kern handelt es sich bei dem Verbot mittelbarer Diskriminierung um eine Beweislastregel, die verhindern soll, dass eine Benachteiligung bestimmter Personen hinter anscheinend neutralen Unterscheidungskriterien »versteckt « wird $^{27}$. Normen und Regelungen, die in der Realität Frauen oder Männer nachteilig treffen, werden als Indiz für eine tatsächliche (»materiale«) Diskriminierung angesehen. Diese ist jedenfalls

23 Dazu s. Osterloh, in Sachs, Grundgesetz-Kommentar, 3. Aufl. 2003, Art. 3 Rn. 272 ff.

24 Das Verbot der mittelbaren Diskriminierung wurde in der europäischen Rechtsprechung vom EuGH entwickelt und findet sich heute ausdrücklich in allen Antidiskriminierungsrichtlinien der EU, u.a. in Art. 2 Abs. 1 RL 76/207/EWG wegen Benachteiligung aufgrund des Geschlechts bei Einstellung und Beschäftigung; zur EuGH-Rechtsprechung s. Krebber, in Calliess/Ruffert (Hg.), Kommentar zu EUV/ EGV, 1999, Art. 141, Rn. 42 ff.

25 Allerdings werden in den Gesetzen von Baden-Württemberg, dem Saarland, Bayern, Niedersachsen und Hessen »abendländische« bzw. »christliche« Zeichen und Kleidungsstücke von dem Verbot ausgenommen; diese Regelungen verstoßen gegen das verfassungsrechtliche Gleichbehandlungsgebot, dazu Baer/Wrase, DÖV 2005, S. 243 (245 ff.).

26 Ein häufiger Fall in Großbritannien, wo viele aus Indien eingewanderte Personen leben. Sikhs sind dort z.B. von der Motorradhelmpflicht ausgenommen und dürfen selbst im Polizeidienst einen Turban tragen, s. Markard/Baer, in Haug/Reimer (Hg.), Politik ums Kopftuch, 2005, S. 151 (157) m. w. Nw.

27 S. Schieck, Differenzierte Gerechtigkeit, 2000, S. 67 f. 
hinsichtlich des Geschlechts unzulässig, da in Art. 3 Abs. 2 S. 2 GG ein Gleichstellungsgebot normiert ist, das gerade auf die gesellschaftliche Wirklichkeit zielt ${ }^{28}$.

Das BVerfG geht in seinen jüngeren Entscheidungen allerdings weiter. Es hat das Verbot der mittelbaren Diskriminierung ausdrücklich auf Art. 3 Abs. 3 GG bezogen, wenn auch nicht im Detail geprüft ${ }^{29}$. Danach wären mittelbare Diskriminierungen grundsätzlich bei allen in Art. 3 Abs. 3 GG genannten Merkmalen gleichheitswidrig, also auch bezüglich Abstammung, »Rasse«, Glauben oder religiöser und politischer Anschauungen ${ }^{30}$. Diese Interpretation des Diskriminierungsverbots ist angesichts des Telos der Norm, Ausgrenzung zu verhindern, ebenso sachgerecht wie sie dem Stand des europäischen Antidiskriminierungsrechts entspricht ${ }^{31}$. Sie trägt zudem der Tatsache Rechnung, dass die nach Art. 3 Abs. 3 GG verpönten Diskriminierungsmerkmale als interdependente, also voneinander abhängige Kategorien auf historisch gewachsene und nachweisbare Benachteiligungen innerhalb der Gesellschaft reagieren, und dass es keinen Menschen gibt, der nur eines dieser »Merkmale« lebt, sondern wir alle uns im Hinblick auf all diese Merkmale unterscheiden. Dieses Phänomen der Interdependenz ist auch mit der Rede von der kombinierten Diskriminierung gemeint ${ }^{32}$. Deshalb darf eine faktische Benachteiligung von moslemischen Frauen, die das Kopftuch tragen, nicht ohne die Faktoren »Glaube «, »Herkunft« und »Ethnizität« gedacht werden, die hier ebenfalls bedeutsam sind.

\section{b) Die mittelbare Diskriminierung im Detail}

Eine mittelbare Diskriminierung liegt vor, wenn eine neutrale Handlung oder Regel eine tatsächliche Benachteiligung in Bezug auf ein bestimmtes Merkmal zur Folge hat. Das wird in drei Schritten festgestellt: (1.) Die Bildung von Vergleichsgruppen ${ }^{33}$, (2.) die Feststellung einer auf ein Merkmal bezogenen unterschiedlichen Betroffenheit und (3.) die Feststellung der Relevanz dieser unterschiedlichen Betroffenheit ${ }^{34}$.

28 So jetzt besonders deutlich: BVerfGE 109, S. 64 (90 f.) - Mutterschutzgesetz; stellvertretend für viele s. Sacksofsky, Das Grundrecht auf Gleichberechtigung, 2. Aufl. 1996, S. 348 f.

29 BVerfGE 97, S. 35 (43f.) - Hamburgisches Ruhegehaltsgesetz; BVerfGE 104, S. 373 (396) -Familiendoppelname; so auch Osterloh, in Sachs, Grundgesetz-Kommentar, Art. 3 Rn. 255; in eine andere Richtung weisend nunmehr jedoch BVerfG, NJW 2005, 2443 - Erziehungszeiten in der Anwaltsversorgung: Schutz vor faktischen Benachteiligungen sei in Art. 3 Abs. 2 GG zu verorten.

30 Die Diskriminierung aufgrund der »sexuellen Orientierung «, die im Europarecht, in Fachgesetzen und im Landesrecht ausdrücklich genannt wird, kann letztlich als Diskriminierung aufgrund des (falschen oder hinsichtlich des Partners oder der Partnerin falschen) Geschlechts verstanden werden.

31 Die auf Art. 13 EGV gestützten Richtlinien 76/207/EWG, 2000/43/EG und 2000/78/EG verbieten die mittelbare Diskriminierung in Bezug auf die Merkmale Geschlecht, »Rasse« und ethnische Herkunft, Religion, Weltanschauung, Behinderung, Alter und sexuelle Ausrichtung.

32 Vgl. dazu die Regelung in $\$ 4$ E-ADG, BT-Drucks. 15/4538.

33 Die Orientierung an Gruppen ist allerdings problematisch, da solche Gruppen in deutlich abgrenzbarer Form nicht existieren. Sinnvoller wäre die Orientierung auf Strukturen der Diskriminierung.

34 S. Schieck, Differenzierte Gerechtigkeit, 2000, S. 65. 
Eine wesentliche Weiche wird im ersten Schritt gestellt. Die Entscheidung für eine bestimmte Vergleichsgruppe präjudiziert sehr häufig, wie der Fall zu entscheiden sein wird. Im Fall des Kopftuchs im Schuldienst lässt sich als Vergleichsgruppe, die von den schul- oder beamtengesetzlichen Verboten der Länder betroffen ist, die Gruppe aller Lehrenden an öffentlichen Schulen bestimmen, die religiöse Symbole oder Kleidungsstücke tragen. Dann ist diese Gruppe relativ klein, und ein Verstoß gegen Gleichheitsrecht wohl nicht gegeben, denn all diese Menschen werden ja gleichermaBen zu bestimmten Verhaltensweisen gezwungen. Der Vergleich betrifft nur die so genannten »Minderheitenreligionen «, und kulturell wird auch »das Problem« entsprechend attributiert. Noch deutlicher wird das, wenn eine Muslima mit all den Frauen verglichen wird, die aus religiösen Gründen eine Kopfbedeckung tragen.

Wird jedoch als Vergleichsgruppe die Gruppe aller Lehrenden unabhängig vom Glauben und unabhängig vom Geschlecht bestimmt, fällt der Vergleich anders aus. Da es in der christlichen Glaubenslehre keinen Verhüllungs- oder Bekleidungszwang für Gläubige gibt, zeigt sich deutlich, wer genau einem Kopfbedeckungsverbot unterfällt. In Deutschland werden dem Islam etwa 3,2 Mio. Gläubige zugerechnet, weit dahinter liegen die Orthodoxen, die Neuapostolische Kirche, die Zeugen Jehovas und die jüdische Gemeinde mit zusammen etwa 1,6 Mio. Glaubensanhängerinnen und -anhängern $^{35}$. In keiner anderen dieser Religionen gibt es eine ähnlich stark vertretene Richtung, die ihren Gläubigen auch im Alltagsleben eine bestimmte Bekleidung vorschreibt. Folglich betreffen die schulgesetzlichen Verbote in der Lebenswirklichkeit weit überwiegend moslemische Frauen, die das Kopftuch tragen. Es liegt also eine faktische Benachteiligung gerade dieser Frauen vor, weil sie als Angehörige eines Geschlechts einer bestimmten Glaubensrichtung angehören.

Im zweiten Schritt der Feststellung mittelbarer Diskriminierung geht es um die Festlegung des relevanten Merkmals. In der deutschen Dogmatik bzw. Rechtswissenschaft besteht die Tendenz, hier ein Merkmal zu betonen, aber die Zusammengehörigkeit mehrerer Merkmale außer Acht zu lassen. Schon die Vergleichsgruppenbestimmung zeigt, dass sowohl Geschlecht als auch Glaube eine Rolle spielen. Daneben muss angesichts der Einwanderungsgeschichte in der Bundesrepublik Deutschland ${ }^{36}$ zudem noch die ethnische (Migrations-)Herkunft berücksichtigt werden. Der größte Teil der Menschen islamischen Glaubens unterliegt einer entsprechenden Zuschreibung als »Ausländerin« oder »fremd«, ist also in einem weiteren Merkmal des Art. 3 Abs. 3 GG berührt.

Im dritten Schritt ist festzustellen, inwieweit die Betroffenheit bezüglich des Merkmals auch tatsächlich bedeutsam oder relevant ist. Da hier gravierende Grenzen in der beruflichen Entwicklung gezogen werden, ist dies bezüglich aller Merkmale der Fall.

35 S. Fischer Weltalmanach 2004, Deutschland S. 215. Die Zahlen stammen aus dem Jahr 2001 und basieren auf Hochrechnungen der letzten Volkszählung. Die zahlenmäßige Erfassung von Moslems ist ein besonderes Problem, da diese nicht offiziell in »Kirchen « oder »Konfessionen « organisiert sind. Es wird daher von der kulturellen Zugehörigkeit ausgegangen. Ausführliche statistische Darstellungen finden sich im Datenarchiv der Forschungsgruppe Weltanschauungen in Deutschland: http://www.fowid.de/nc/datenarchiv/.

36 Dazu s. Langenfeld, RdJB 2004, S. 4. 


\section{c) Rechtfertigung durch das verfassungsrechtliche Neutralitätsgebot?}

Im Geltungsbereich des Verbotes mittelbarer Diskriminierung wird nun zunächst, prima facie, vermutet, dass die diskriminierende Wirkung auf einer Diskriminierungshandlung beruht. Diese Vermutung kann jedoch widerlegt werden, wenn sachliche Gründe dafür sprechen, ein neutrales Kriterium - hier also das Verbot religiöser Kleidungsstücke für Lehrpersonen - zu verwenden, und wenn die Regelung aus eben diesen sachlichen Gründen verhältnismäßig ist ${ }^{37}$.

Ein sachlicher Grund könnte darin liegen, dass Lehrpersonen an öffentlichen Schulen, die sichtbar religiöse Symbole tragen, gegen das staatliche Neutralitätsgebot in Glaubensfragen verstoßen. Nun ist »Neutralität« eben kein feststehender Begriff, sondern ein offenes Konzept, das mit unterschiedlichen Inhalten gefüllt werden kann. Das BVerfG hat schon zur christlichen Gemeinschaftsschule und zum Gemeinschaftsgebet ausgeführt, dass die religiös-weltanschauliche Neutralität im Grundgesetz keinen strikten Ausschluss aller religiösen Bezüge aus dem staatlichen Bereich verlangt. Der Staat dürfe lediglich nicht »Position« zugunsten einer bestimmten Glaubensrichtung beziehen ${ }^{38}$. Das tut er dann nicht, wenn er die Glaubensbekundung der Lehrerin, die mit Kopftuch unterrichtet, als erkennbaren Ausdruck ihrer eigenen Persönlichkeit toleriert. Diese Form des individuellen Ausdrucks ist insbesondere mit der staatlich angeordneten, also zwangsweisen Anbringung eines Kreuzes oder Kruzifixes im Klassenraum nicht vergleichbar ${ }^{39}$.

Die offene Neutralität des Grundgesetzes schreibt dem Staat allerdings auch nicht vor, religiöse Bekleidung von Lehrkräften generell zu tolerieren. Religiöse Bezüge in Schule und Unterricht können, so meint das Gericht, den in Neutralität zu erfüllenden staatlichen Erziehungsauftrag, das elterliche Erziehungsrecht und die negative Glaubensfreiheit der Schülerinnen und Schüler beeinträchtigen. Es liege zwar keine konkrete, aber zumindest eine potentielle oder abstrakte Gefahr für diese Rechte vor ${ }^{40}$. Nach Ansicht des BVerfG darf der Staat also religiöse Bekundungen und Zeichen im öffentlichen Lehrdienst verbieten, weil davon - möglicherweise - Irritationen und Konflikte ausgehen könnten. Er kann sich aber auch auf einen toleranten Umgang mit diesen Symbolen einlassen. Er hat also die Wahl zwischen einer strengen Neutralitätsund einer Toleranzlösung ${ }^{41}$.

In den Bundesländern, die eine Toleranzlösung avisieren, besteht allenfalls Bedarf nach einer Regelung für den Umgang mit Konflikten, sollten diese denn wirklich auftreten. Für die Bundesländer jedoch, die eine strenge Lösung bevorzugen, also ihrem Lehrpersonal das Tragen religiöser Kleidungsstücke im Unterricht verbieten wollen, hat das BVerfG zwei weitere verfassungsrechtliche Bedingungen aufgestellt: (1.) Das Verbot muss, da es sich um eine grundrechtsrelevante und folglich wesentliche Frage handelt, in einem Parlamentsgesetz beschlossen werden. Das soll die Voraussehbar-

37 Schieck, Differenzierte Gerechtigkeit, 2000, S. 64f.

38 BVerfGE 108, S. 282 (300).

39 BVerfGE 108, S. 282 (302).

40 BVerfGE 108, S. 282 (303).

41 S. Baer/Wrase, DÖV 2005, S. 243 (245). 
keit einer Maßnahme und die demokratische Legitimation sichern ${ }^{42}$. (2.) Das Verbotsgesetz muss Angehörige unterschiedlicher Religionsgemeinschaften strikt gleich behandeln ${ }^{43}$. Auf die Suche nach einem Kompromiss zwischen den unterschiedlichen rechtlich anerkannten Interessen (»praktische Konkordanz«) verzichtet das Gericht ebenso wie auf die oben angedeutete komplexe Prüfung der ineinander greifenden Rechte und ihrer Rechtfertigungen. Die Gleichzeitigkeit der Betrachtung unterschiedlicher rechtlicher Interessen wird gerichtlich nicht verarbeitet, sondern pragmatisch delegiert. Das Bundesverfassungsgericht möchte sich offenkundig gerade nicht zur letzten Instanz aufspielen, und juristisch determinierte gerichtliche Entscheidungen geben Zwischenlösungen keinen Raum ${ }^{44}$. Was aber bedeutet es nun für die Landesgesetzgeber, Lehrkräfte »strikt gleich« zu behandeln?

\section{d) Ein Kopftuchverbot zum Schutz der Gleichberechtigung?}

Gesellschaftspolitisch geht es weder in Baden-Württemberg noch andernorts um ein Verbot der Kippa, oder des Turbans, sondern um das islamische Kopftuch. Wer nun das Kopftuch als solches verbieten, andere religiöse Kleidungsstücke allerdings weiterhin zulassen will, muss eine konkrete Gefährdung gerade durch dieses Symbol darlegen. Eine solche Gefährdung läge vor, wenn das Kopftuch eine per se verfassungsfeindliche Bedeutung hätte, oder aber, wenn es eine besonders negative Wirkung auf die Schulkinder ausübte. Und genau das wird vielfach behauptet: Das Kopftuch sei über seine Funktion als religiöses Symbol hinaus ein Zeichen der minderen Stellung der Frau in der Gesellschaft sowie Ausdruck einer fundamentalistischen, kämpferischen Stellungnahme für ein islamisch-theokratisches Staatswesen ${ }^{45}$. Im Minderheitsvotum der Verfassungsrichter heißt es ausdrücklich: »Das Kopftuch, getragen als kompromisslose Erfüllung eines von der Beschwerdeführerin [Frau Ludin] angenommenen islamischen Verhüllungsgebotes der Frau, steht gegenwärtig für viele Menschen innerhalb und außerhalb der islamischen Religionsgemeinschaft für eine religiöse begründete kulturpolitische Aussage, insbesondere das Verhältnis der Geschlechter betreffend [...]. Immerhin wurzelt auch nach Meinung wichtiger Kommentatoren des Korans das Gebot der Verhüllung der Frau - unabhängig von der Frage, ob es überhaupt ein striktes Gebot in diese Richtung gibt - in der Notwendigkeit, die Frau in ihrer dem Mann dienenden Rolle zu halten. Diese Unterscheidung zwischen Mann und Frau steht dem Wertebild des Art. 3 Abs. 2 GG fern. [...] Schon ein weiterer Schritt hin zur gänzlichen Verhüllung des Gesichts, der ebenfalls in der islamischen Glaubensgemeinschaft praktiziert wird, könnte aus deutschem Verfas-

42 BVerfGE 108, S. 282 (311).

43 BVerfGE 108, S. 282 (313).

44 Die in der Literatur vorgeschlagene Widerspruchslösung dürfte in der Praxis zu erheblichen Schwierigkeiten führen. Außerdem wäre es wohl kaum angemessen, dass die Lehrerin im Einzelfall von der »Toleranz« ihrer Schulkinder und deren Eltern abhängig sein soll. Berlin hat die Widerspruchs-Regelung jetzt für den Kita-Bereich eingeführt. Es bleibt abzuwarten, ob sich dieser Weg bewährt, s. Baer/Wrase, DÖV 2005, S. 243 (247).

45 So u.a. die Gesetzesbegründung des Landes Baden-Württemberg, s. LT-Drs. Bad-Württ. 13/2793, S. 7. 
sungsverständnis heraus als unvereinbar mit der Würde des Menschen angesehen werden: Der freie Mensch zeigt dem anderen sein Antlitz $\aleph^{46}$.

Es wäre interessant, dem hier zugrunde liegenden kulturhegemonialen Vorverständnis genauer nachzugehen. Verfassungsrechtlich ist vorrangig zu klären, ob das Kopftuch tatsächlich dem Bild der gleichberechtigten Frau in unserer Gesellschaft widerspricht, wie es ja auch Alice Schwarzer und andere behaupten. Ist dies der Fall, so lassen sich die durch ein Verbot erzeugten Eingriffe in die Grundrechte der Betroffenen unter Berufung auf Art. 3 Abs. 2 GG als kollidierendes Verfassungsrecht rechtfertigen $^{47}$. Dies gilt insbesondere, da Art. 3 Abs. 2 GG einen Auftrag zur tatsächlichen Gleichstellung der Geschlechter beinhaltet und dem Staat diesbezüglich einen Schutzauftrag erteilt ${ }^{48}$. In der Frage nach dem Bedeutungsgehalt des islamischen Kopftuchs liegt offensichtlich der Schlüssel zur »Lösung « des verfassungsrechtlichen Problems.

\section{e) Die (Be-)Deutung(en) des islamischen Kopftuchs}

Wer nach der Bedeutung des Kopftuchs sucht, wird allerdings ganz unterschiedliche Antworten erhalten. Eine allgemeine islamische Glaubensregel, die Frauen das Tragen des Kopftuchs oder auch des Schleiers vorschreibt, gibt es nicht. Dies ist vor allem der Tatsache geschuldet, dass der Islam keine Einheitskirche ist, sondern verschiedene Lehren nebeneinander stehen ${ }^{49}$. »Das « Kopftuch ist daher zunächst einmal ein profanes Stück Stoff, ein Kleidungsstück. Erst durch die Verbindung mit bestimmten religiösen Bedeutungen bekommt es die Wirkung eines Symbols. Ein Symbol ist ein Sinnbild und wird in einem sozialen Kommunikationsprozess $»$ konstruiert $~^{50}$. Ein Symbol ist, wie Janbernd Oebbecke darlegt, »inhaltlich nicht eindeutig zu bestimmen, da es als prinzipiell unendlich interpretierbare Variable in Abhängigkeit vom jeweiligen Kontext mit seinen möglichen Inhalten und seinen möglichen Interpreten korreliert und so stets auch neue Bedeutungen erhalten kann ${ }^{51}$. Mit anderen Worten: Was man an (Be-)Deutungen in einem Symbol erkennt, ist eine Frage der eigenen Interpretation: Diese Interpretation ist abhängig vom sozial-kulturellen Kontext, in dem das Symbol erkennbar wird, und abhängig von der Erfahrungswelt und den Anschauungen der Interpretierenden. Eine überindividuelle Symbolbedeutung entwickelt sich erst in einem Kommunikationsprozess. An diesem sind allerdings keineswegs alle gleichermaßen beteiligt. Wie insbesondere in kritischen Debatten um diskursive Legitimationsmodelle allgemeinverbindlicher Entscheidungen immer wieder betont wird, ist der Diskurs kei-

46 BVerfGE 108, 282 (333f.).

47 Grundrechte werden hier als verfassungsrechtliche Wertentscheidungen verstanden; Dreier, in: ders., Grundgesetz-Kommentar, Bd. I, 2. Aufl. 2004, Vorb. Rn. 140. In einer Grundrechtsdogmatik kollidierender Freiheits- und Gleichheitsrechte ist dieser Wertbezug allerdings nicht zwingend.

48 U.a. BVerfGE 82, S. 191 (207) - Nachtarbeitsverbot; BVerfGE 89, 276 (285) Einstellungsdiskriminierung; BVerfGE 109, S. 64 (89) - Mutterschutzgesetz.

49 Baer/Wrase, Jus 2003, S. 1162 (1163).

50 Oebbecke, Das »islamische Kopftucht« als Symbol, in Festschrift für Rüffner, S. 593 (594f.).

51 S. Oebbecke, ebenda, S. 594. 
neswegs herrschaftsfrei und auch nicht allen gleichermaßen zugänglich. Es ist daher auch keinesfalls undenkbar, dass die von Betrachtenden wahrgenommene Bedeutung eines Symbols von der Bedeutung abweicht, die der Produzent bzw. die Produzentin mit dem Symbol verbindet ${ }^{52}$. Vielmehr ist diese Deutungsdiskrepanz ein typisches Kennzeichen von Diskriminierungsphänomenen, die ja darauf beruhen, dass Menschen Ausgrenzungen erfahren, die sie an Deutungsprozessen nicht teilhaben lassen. So stellt sich bei der Betrachtung von Symbolen nicht schlicht die Frage, was sie bedeuten, sondern immer die Frage, wessen Sichtweise oder (Be-)Deutung entscheidend wirkt.

\section{f) Das Verhüllungsgebot}

Es mag sein, dass viele Menschen in Deutschland das Kopftuch mit Frauenverachtung gleichsetzen, wenn es von Frauen getragen wird, die sie anderen Kulturkreisen als dem eigenen zuordnen. Angesichts des Rechts zur Selbstbestimmung gerade in religiösen Fragen scheint jedoch wichtiger die Frage, welche Bedeutung das Kopftuch für die Frauen hat, die es selbst tragen.

Weltweit trägt die große Mehrheit der muslimischen Frauen kein Kopftuch. Die Auffassung, dass Frauen in der Öffentlichkeit ihren Kopf und (teilweise) auch ihr Gesicht $\mathrm{zu}$ bedecken haben, ist allerdings in einzelnen islamischen Glaubensrichtungen, Staaten oder Kulturen verbreitet ${ }^{53}$. Sie stützt sich auf einige Suren des Korans über die Frauen des Propheten Mohammed ${ }^{54}$. Ein striktes Verhüllungsgebot ist auch darin allerdings nicht enthalten. Ein solches findet sich erst in der Sunna und späteren Gelehrtensammlungen (Hadithen), in denen Frauen zum Teil deutlich sexualisiert werden und ihnen eine untergeordnete Rolle zugewiesen wird (die Frau als Aurah = Mängelwesen $)^{55}$. Die Frau soll den Blicken des Mannes entzogen werden. Eine Frau, die sich in der Öffentlichkeit nicht verhüllt, wird als unrein und sexuell minderwertig angesehen.

Dementsprechend findet sich in islamistischen Ländern wie Saudi-Arabien, Iran und anderen Golfstaaten, die diese Regel zwingend durchsetzen, nicht nur ein staatlicher oder faktischer Verhüllungszwang, sondern eine deutliche Separierung und Unterordnung von Frauen im öffentlichen und im privaten Leben ${ }^{56}$. Andere Staaten, die sich zum islamischen Recht bekennen, folgen einer gemäßigten Interpretation des Koran bzw. der Sunna, die Frauen nach wie vor deutlich benachteiligt, ihnen aber gewisse Freiräume lässt. Sie leiten aus einem koranischen Gebot ab, dass man seinen Verstand gebrauchen muss, um eine zeitgemäße Auslegung der überlieferten Regeln zu fin$\operatorname{den}^{57}$. Die Gesamtheit der islamischen Lebensregeln, die Scharia, wird ebenfalls sehr

52 Oebbecke, ebenda, S. 595.

53 S. Rohe, Der Islam - Alltagskonflikte und Lösungen, 2. Aufl. 2001, S. 145.

54 S. Oestreich, Der Kopftuch-Streit, 2004, S. 15 ff.; Knieps, Geschichte der Verschleierung der Frauen im Islam, 1993.

$55 \mathrm{Vgl}$. Knieps, ebenda, S. 262 ff.

56 S. Oestreich, Der Kopftuch-Streit, 2004, S. $22 \mathrm{f}$.

57 Oestreich, ebenda, S. 20; wie eine solche »zeitgemäße« Intrepretation von islamischem Recht aussehen kann, zeigt bspw. die Verbesserung der Frauenrechte im ägyptischen Eherecht, s. Denker, Die Wiedereinführung des Hul’ und die Stärkung der Frauenrechte, in Tellenbach/Hanstein (Hg.), Beiträge zum islamischen Recht IV, 2004, S. 125. 
unterschiedlich interpretiert. Viele Muslime sehen darin überhaupt nur einen ethischen Leitfaden, der die persönliche Lebensführung beeinflusst. Fundamentalisten dagegen erheben die strenge Auslegung der Scharia zum Gesetz ${ }^{58}$.

In Deutschland vertreten islamische Gemeinschaften entsprechend unterschiedliche Positionen. Die meisten Muslime leben hier in der zweiten oder dritten Generation migrierender Familien. Der überwiegende Teil kam in den sechziger und siebziger Jahren aus der Türkei, als in der Bundesrepublik ein Mangel an Arbeitskräften in den unqualifizierten und schlechter bezahlten Berufen herrschte. Heute befinden sich viele (nach wie vor) in einer Diasporasituation, die als »Parallelgesellschaft « zunehmend auch öffentlich problematisiert wird. Integration erfolgt nicht immer und keinesfalls problemlos. Für die teils als zunehmend konstatierte Desintegration sind neben veränderten ökonomischen Bedingungen maßgeblich auch politische und gesellschaftliche Versäumnisse verantwortlich ${ }^{59}$.

In vielen muslimischen Familien in Deutschland sind Frauen patriarchalischen Zwängen ausgesetzt. Anders als in anderen patriarchalen Familien eröffnen mangelnde Integration und eine daraus resultierende feste Einordnung in die muslimische Community wenig Chancen, sich von diesen Strukturen zu emanzipieren.

Diese spezifische Szenerie in der deutschen Diaspora spielt auch für die Frage nach der Bedeutung des Kopftuchs eine wichtige Rolle ${ }^{60}$. Neuere sozialwissenschaftliche Studien, u.a. von Nökel (2002), Klinkhammer (2000), Karagasoglu (2000) und Rommelspacher (2002), die sich mit Kopftuchträgerinnen bzw. mit moslemischen Frauen der dritten Generation in Deutschland befassen, zeichnen ein differenziertes Bild von den Ansichten und Motiven dieser Frauen ${ }^{61}$. Heide Oestreich fasst die Ergebnisse in ihrem Buch über »den Kopftuch-Streit« so zusammen: Junge Frauen tragen das Kopftuch nicht aus traditionellen Gründen, sondern haben es neu entdeckt und für sich mit Sinn gefüllt. Es gibt einige junge Frauen, deren Eltern wollten, dass sie das Tuch tragen, andere haben es eher gegen die kemalistisch beeinflussten Eltern durchsetzen müssen. Die Mädchen tragen ihr Kopftuch - den Türban - ab Beginn ihrer Pubertät. Diesen Schritt begreifen sie als eine Art Initiation, mit der sie sich offensiv zur islamischen Religion und Kultur bekennen. Das Kopftuch ist für sie Ausdruck ihres Selbstbildnisses als islamische Frauen. Damit wird es zu einem Zeichen gesellschaftlicher und religiöser Identität, einem »Identitätsmarker « ${ }^{62}$.

Derartige Befunde sind allerdings auch kritisiert worden, wie von Johannes Kandel und Christoph Müller-Hofstede ${ }^{63}$. Sie weisen darauf hin, dass nur sehr kleine, spezifische Gruppen von Muslimminen, in der Regel akademisch gebildete Frauen, unter-

58 Oestreich, ebenda, S. 20.

59 S. Langenfeld, RdJB 2004, S. 4 (8).

60 S. Oestreich, Der Kopftuch-Streit, 2004, S. 131.

61 Insb. Nökel, Die Töchter der Gastarbeiter und der Islam, 2002; Klinkhammer, Moderne Formen islamischer Lebensführung, 2000; Karakasoglu, Muslimische Religiosität und Erziehungsvorstellungen, 2000; Rommelspacher, Anerkennung und Ausgrenzung, 2002.

62 Oestreich, Der Kopftuch-Streit, 2004, S. 144.

63 Kandel/Müller-Hofstede, Buchrezension zu »Heide Oestreich: Der Kopftuchstreit. Das Abendland und ein Quadratmeter Islam«, unter www.fes-online-akademie.de. 
sucht wurden. Es sei fraglich, ob das Kopftuch für diese Frauen wirklich Ausdruck von Eigenständigkeit sei. Vielmehr lasse sich die Persistenz eines geschlechtersegregierten, männerdominierten Weltbildes in weiten Teilen des organisierten islamischen Milieus nicht bestreiten. Die frauenfeindliche Bedeutung des Kopftuchs sei historisch gewachsen und bis heute sowohl in islamischen Staaten als auch in der Diaspora ungebrochen. Es gebe auch keine öffentliche Kritik engagierter islamischer Frauengruppen, die das Kopftuch befürworten, sich gleichzeitig aber gegen das entwürdigende Frauenbild der vier Rechtsschulen und zahlloser Hadithe wenden ${ }^{64}$.

Die Kritik von Kandel und Müller-Hofstede sollte nicht mit leichter Hand zurückgewiesen werden. In der Tat ist das Bild, das Heide Oestreich und Sigrid Nökel von den jungen Kopftuchträgerinnen (»Neo-Muslimas«) zeichnen, vielleicht ein wenig zu optimistisch. Es ist nicht so, dass die befragten Frauen das Kopftuch lediglich dazu benutzen, um damit den Restriktionen ihrer Eltern oder Ehemänner $» z u$ kontern $\aleph^{65}$. Vielmehr bejahen die meisten Muslimminen in den Interviews ein klassisch-traditionelles Weiblichkeitsideal, das für die Frau grundsätzlich die Rolle der »Haus-«Frau und Mutter vorsieh $t^{66}$. Dabei darf aber nicht vergessen werden, dass die meisten Muslimminen durch ihr soziales Umfeld innerhalb der Diaspora ohne jede Alternative auf diese Position festgelegt sind. Zudem ist diese Position ja auch im Christentum und in zeitgenössischen Debatten um Geschlechterrollen keineswegs unbekannt.

Das Kopftuch ist auch in den Augen der »Neo-Muslimas « kein Ausdruck einer emanzipierten Lebensweise im »westlichen « Sinn. Das Kopftuch bietet Frauen jedoch die Möglichkeit, »Loyalität« zur eigenen Kultur zu bekunden und dennoch Freiheitsräume anzustreben ${ }^{67}$. Es ist also zu weit gehend, den Studien von Nökel, Klinkhammer, Karagsoglu und Rommelspacher pauschal jede Bedeutung abzusprechen und darauf zu verweisen, dass auch innerhalb der Einwandererfamilien ein geschlechtersegregiertes und diskriminierendes Rollenverständnis vorherrschend sei $^{68}$. Die Studien verdeutlichen vielmehr, dass die jungen Muslimminen sich dieser Situation bewusst gegenüber sehen und sich deshalb von vornherein nur in begrenztem Umfang Freiräume schaffen können. Ein Mittel dazu kann auch das bewusste Tragen des Kopftuchs sein. Ein frauenfeindliches Symbol ist es damit per se in einem rechtlich relevanten Sinne nicht $^{69}$. Unter Bezug auf die Studie von Yasemin Karakasoglu hat das BVerfG jedenfalls für das Verfassungsrecht mit Recht festgestellt, die Deutung des Kopftuchs dürfe »nicht auf ein Zeichen gesellschaftlicher Unterdrückung der Frau verkürzt werden $\ll^{70}$.

64 Kandel/Müller-Hofstede, ebenda, S. 2.

65 So Oestreich, Der Kopftuch-Streit, 2004, S. 144.

66 Vgl. etwa Klinkhammer, Moderne Formen islamischer Lebensführung, 2000, S. 276.

67 So bereits Baer/Wrase, JuS 2003, S. 1162 (1165).

68 So Kandel/ Müller-Hofstede, aaO (o. Fußn. 62), S. 2 f.

69 Britz, KJ 2003, S. 95 (99).

70 BVerfGE 108, S. 282 (305). 


\section{g) Art. 3 Abs. 2 GG und die »Vorbildfunktion« der Lehrerin}

Nun ließe sich argumentieren, das Kopftuch stehe zwar für die Trägerin vielleicht nicht per se für Unterdrückung von Frauen, aber doch für ein sehr konservatives Frauenbild und werde so dem Anspruch der staatlichen Schule nicht gerecht, den Gleichstellungsauftrag des Grundgesetzes bzw. der Landesverfassungen zu verwirklichen. Auch dieses Argument greift gesellschaftspolitisch, verfassungsrechtlich jedoch nicht. Die Rechtsprechung des BVerfG hat vielmehr betont, dass Gleichstellung bedeute, niemanden auf tradierte Rollen zu fixieren, aber auch niemandem zu verbieten, diesen anzuhängen. Die Pflicht des Staates zur Förderunge der Gleichstellung bedeutet gerade nicht, dass eine Frau gegen ihren Willen gezwungen werden kann, einem bestimmten Bild einer (»westlich«) emanzipierten Frau zu entsprechen. Auch an die Übernahme von traditionellen Geschlechterrollen dürfen keine ungerechtfertigten Nachteile geknüpft werden ${ }^{71}$. Eine Lehrerin oder Lehramtsanwärterin kann folglich nicht um ihrer eigenen Emanzipation willen gezwungen werden, das Kopftuch abzulegen $^{72}$. Sie kann auch nicht zu einer bestimmten Kleidung gezwungen werden, um ein ganz bestimmtes Vorbild für Schülerinnen und Schüler zu sein ${ }^{73}$. So darf der Staat von seinen Beamtinnen und Beamten nicht erwarten, dass sie außerhalb ihres Dienstes ein Leben führen, das in jeder Hinsicht einem gleichberechtigten, emanzipatorischen Idealbild entspricht. Wie Gabriele Britz mit Recht betont, müssten unter Anlegung eines solchen Maßstabs auch die zahlreichen halbtags beschäftigten (christlichen) Lehrerinnen vom Schuldienst ausgeschlossen werden, »die auf weitgehende Karrierechancen verzichten, um dem (christlichen) Gatten und Familienvater die Vollzeitkarriere zu ermöglichen $\aleph^{74}$. Hinzufügen könnte man, dass manchmal auch männliche Staatsangestellte, ja selbst Spitzenbeamte, sich nach wie vor offen für eine feste »traditionelle« Rollenverteilung der Geschlechter aussprechen, die Frauen benachteiligt ${ }^{75}$ - ohne dass jemals jemand ihre Entlassung gefordert hätte.

Welche Haltung aber darf der Staat von seinen Beamtinnen und Beamten gegenüber den Zielen der Gleichstellung nach Art. 3 Abs. 2 GG verlangen? In seiner Entscheidung Vogt gegen Deutschland hat der Europäische Gerichtshof für Menschenrechte (EGMR) entschieden, dass eine Deutsch- und Französischlehrerin nicht allein wegen ihrer Mitgliedschaft in einer als verfassungsfeindlich eingestuften Partei (DKP) aus dem Staatsdienst entlassen werden darf ${ }^{76}$. Es müsse vielmehr die tatsächliche Gefahr

71 S. Britz, KJ 2003, S. 94 (99); Sacksofsky, Das Grundrecht auf Gleichberechtigung, 2. Aufl. 1996, S. 352f.; vgl. Baer, Würde oder Gleichheit, 1995, S. $239 \mathrm{ff}$.

72 Vgl. Britz, KJ 2003, S. 94 (99).

73 S. etwa Hufen, NVwZ 2004, S. 575 (576); abw.M. BVerfGE 108, S. 282 (330f.).

74 Britz, KJ 2003, S. 94 (100).

75 Im Bundestagswahlkampf 2005 prominent geworden ist das Beispiel des Heidelberger Rechtsprofessors Paul Kirchhof, der sogar als zukünftiger Finanzminister gehandelt wurde. Kirchhof hatte u.a. gesagt: "Die Mutter macht in ihrer Familie Karriere, die nicht Macht, sondern Freundschaft verheißt, nicht Geld, sondern Glück bringt.«, s. Familienbande - Merkel und die Kirchhof-Töchter, Spiegel-Online - 05. September 2005, http://www.spiegel.de/politik/deutschland/.

76 EGMR NJW 1996, S.375. 
einer unangemessenen Einflussnahme oder Indoktrination bestehen. Außerdem dürfen Äußerungen der Lehrerin nicht ignoriert werden, wenn sie sich im Dienst positiv zu den Werten der Verfassung bekennt ${ }^{77}$. Diese Grundsätze lassen sich auf den KopftuchFall übertragen. Die Lehramtsbewerberin muss eine Gewähr dafür bieten, dass sie mit anderen religiösen Auffassungen tolerant umgeht, sich nach außen positiv gegenüber dem Ziel der Gleichberechtigung einsetzt und die Schulkinder nicht im Sinne einer bestimmten (schon gar nicht: fundamentalistischen) Auffassung beeinflusst oder indoktriniert. Das wäre beispielsweise zu verneinen, wenn eine Lehrerin Frauen, die kein Kopftuch tragen, als »unrein « erklärt oder in der Schule diskriminierende Abgrenzungen zwischen Menschen verschiedener Glaubensrichtungen oder verschiedenen Geschlechts vornimmt ${ }^{78}$.

Das Kopftuch allein rechtfertigt den Vorwurf der Indoktrination allerdings nicht. Für eine nachhaltige Beeinflussung von Schülerinnen und Schülern durch den Anblick des Kopftuchs ihrer Lehrerin fehlt es an konkreten Anhaltspunkten ${ }^{79}$. Schulkinder sind durchaus in der Lage, das Tragen des Kopftuchs als Ausdruck der individuellen Persönlichkeit der Lehrerin zu begreifen und zu akzeptieren, oder gerade das zu lernen ${ }^{80}$. Auch für eine Beeinträchtigung des »Schulfriedens « gerade durch das Kopftuch fehlt es an Indizien, denn bisherige Erfahrungen mit Kopftuchträgerinnen im Schuldienst geben für eine solche Gefahrenprognose keinen Anlass ${ }^{81}$.

In der Literatur und auch in den Begründungen der Landesgesetze wird demgegenüber die Auffassung vertreten, das Kopftuch werde aus Sicht der Schülerinnen und Schüler, der Eltern und der Öffentlichkeit zwingend als Symbol der Ungleichbehandlung, der Frauendiskriminierung und einer orthodoxen und missionarischen Deutung der islamischen Religion wahrgenommen ${ }^{82}$. Dies sei als »objektiver Empfängerhorizont « der Bewertung des Kopftuchs zugrunde zu legen. Gerade das ist allerdings eben die einseitige Deutung, die dem Staat aufgrund des religiösen Toleranzgebotes versagt ist. Es sind vielmehr alle Deutungsmöglichkeiten zu berücksichtigen ${ }^{83}$.

Muslimische Frauen in Deutschland dürfen nicht pauschal für einen fundamentalistischen Islam in »Gesinnungshaft« genommen werden. Religiöse Symbole dürfen nicht allgemein mit Inhalten identifiziert werden, die nur von bestimmten Gruppen innerhalb einer größeren (heterogenen) Glaubensrichtung vertreten werden. Genauso wenig wäre es gerechtfertigt, das christliche Kreuz pauschal mit streitbaren Deutungen innerhalb des Christentums zu verknüpfen, beispielsweise der Haltung der katholischen Kirche zur Priesterschaft von Frauen oder christlich-fundamentalistischen Ansichten, wie sie neuerdings u.a. in Teilen der Vereinigten Staaten Konjunktur haben.

77 EGMR NJW 1996, S 355 (380).

78 So bereits Baer/Wrase, JuS 2003, 1162 (1166).

79 BVerfGE 108, S. 282 (306f.).

80 S. auch Laskowski, KJ 2003, S. 420 (433).

81 S. Alan/Steuten, ZRP 1999, S. 209 (214).

82 S. gleichlautend Saarl. LT-Drs. 12/1072, S. 4; LT-Drs. Bad-Württ. 13/2793, S. 7; Bay. LTDrs. 15/368, S. 4; Hess. LT-Drs. 16/1897, S. 4; aus der Literatur u.a. Hufen, NVwZ 2004, S. 575 ( 576); Battis/Bultmann, JZ 2004, S. 581 (583).

83 BVerfGE 108, S. 282 (305). 
Dies würde vielen Menschen christlichen Glaubens Unrecht tun, die das Kreuz gerade nicht mit diesen Inhalten verbinden. Von Schülerinnen und Schülern darf ebenso wie von ihren Eltern ein gewisses Maß an gelebter Toleranz erwartet werden, zu dem gerade die Schule beitragen soll. Schule ist nicht zuletzt ein Ort der Kommunikation, in der eine Lehrerin die Möglichkeit hat, ihre persönliche Haltung zu verdeutlichen und eventuelle Missverständnisse auszuräumen ${ }^{84}$.

Eine Sonderbehandlung bestimmter Glaubensrichtungen oder eine Unterteilung in »problematische « und »unproblematische« Symbole ist verfassungsrechtlich nicht zu rechtfertigen ${ }^{85}$. Das gilt auch in Gebieten, in denen der Anteil von Schülerinnen und Schülern muslimischen Glaubens besonders hoch ist. Es wird argumentiert, islamische Organisationen, die von Muslimas das Tragen des Kopftuches fordern, könnten sich durch die Zulassung des Kopftuchs an öffentlichen Schulen bestärkt fühlen, und die Lehrerin mit Kopftuch sei eine Etappe zur »Eroberung islamisierter Räume ${ }^{86}$ oder trage zur Verbreitung eines fundamentalistischen Islam in Deutschland und Mitteleuropa bei ${ }^{87}$. Verkannt wird hier jedoch, dass Prozesse der Abgrenzung und Desintegration gerade innerhalb der muslimischen Minderheit in Deutschland also außerhalb öffentlicher Räume ablaufen und gerade durch einen Ausschluss von Kopftuchträgerinnen von öffentlichen Ämtern verstärkt werden ${ }^{88}$.

Wenn eine muslimische Frau trotz schlechter Startbedingungen in Deutschland nach Schulausbildung und Abitur ein Lehramtsstudium erfolgreich abschließt und dann an einer Schule als verbeamtete Lehrerin unterrichtet, sollte das in den heutigen Verhältnissen als Zeichen für eine gelungene Integration bewertet werden ${ }^{89}$. Diese Lehrerin kann auch wenn sie ein Kopftuch trägt - für junge Muslimminen ein positives Vorbild sein, selbst einen höheren Bildungsabschluss anzustreben und sich so von ihrer nicht selten integrationsfeindlichen und patriarchalischen Umgebung zu emanzipieren. Auch das sollte bedacht werden, bevor das Kopftuch per se als frauenfeindliches Symbol verdammt wird.

\section{Fazit}

Art. 3 Abs. 2, 3 GG streitet also - entgegen der Meinung vieler - grundsätzlich gegen ein Verbot und für eine Zulassung des Kopftuchs im staatlichen Schuldienst. Verbote von religiösen Kleidungsstücken und Symbolen, wie sie in neuen Ländergesetzen vorgesehen sind, sind eine mittelbare Diskriminierung von Frauen, die in der Diasporasituation in Mitteleuropa zudem einer »fremden « Religion und Ethnizität zugehören. Es sind daher mehrere Diskriminierungsmerkmale des Art. 3 Abs. 3 GG betroffen, was häufig übersehen wird.

84 Baer/Wrase, DÖV 2005, S. 243 (248).

85 Baer/Wrase, DÖV 2005, S. 243 (248 ff.); ebenso jetzt: VG Stuttgart VB/BW 2006, 400 f.

86 Kandel/Müller-Hofstede, aaO (Fußn. 62), S. 3.

87 Schwarzer, in: Haug/Reimer (Hg.), Politik ums Kopftuch, 2005, S. 32 ff.

88 So war es nicht Frau Ludin, die mit ihrer Klage auf Einstellung in den Schuldienst öffentliches Aufsehen erregte, sondern die damalige Ministerin Schavan und Publizist(inn)en wie Alice Schwarzer, die Öffentlichkeit herstellten, dazu Zuck, NJW 1999, S. 2948f.

89 Vgl. Debus, NVwZ 2001, S. 1355 (1359). 
Überzeugende Gründe, die ein Kopftuchverbot rechtfertigen können, gibt es nicht. Höchstens ist im Sinne des BVerfG an ein Verbot aller religiösen Kleidungsstücke und Symbole zu denken, da sie ein besonderes Potenzial haben, an den Schulen Ablehnung und Konflikte hervorzurufen. Dann müssen aber alle Glaubensrichtungen strikt gleich behandelt werden. Sondergesetze, die sich faktisch ausschließlich gegen das islamische Kopftuch richten, sind verfassungswidrig.

Die entscheidende Folge eines Kopftuchverbots liegt darin, muslimische Frauen von einem wichtigen Berufsfeld auszuschließen. Dies betrifft Frauen, die es in der Diasporasituation in Deutschland ohnehin schwer haben, sich gegen geschlechtersegregierende und patriarchale Zwänge innerhalb der Einwanderungsgesellschaft durchzusetzen. Dies gilt insbesondere, da sie sich auch in der Mehrheitsgesellschaft kulturell kaum verorten können und von dorther eher Ablehnung als Integration und Aufnahme erfahren ${ }^{90}$.

Auf der anderen Seite kann durch ein Kopftuchverbot und die daraus resultierende Ausgrenzung von Muslimminen aus dem staatlichen Schuldienst nur wenig bis gar nichts für die Gleichstellung gewonnen werden.

90 Britz, KJ 2003, S. 95 (»doppelte Fremdheit«). 